\title{
Absolute and relative extrema, the mean value theorem and the inverse function theorem for analytic functions on a Levi-Civita field
}

\author{
Khodr Shamseddine
}

\begin{abstract}
The proofs of the extreme value theorem, the mean value theorem and the inverse function theorem for analytic functions on the Levi-Civita field will be presented. After reviewing convergence criteria for power series $[\mathbf{1 5}]$, we review their analytical properties $[\mathbf{1 8}, \mathbf{2 0}]$. Then we derive necessary and sufficient conditions for the existence of relative extrema for analytic functions and use that as well as the proof of the intermediate value theorem [20] to prove the extreme value theorem and the mean value theorem. We then complete the study of analytic functions by proving the inverse function theorem. Altogether, we show that analytic functions on the Levi-Civita field have similar smoothness properties to those of real analytic functions.
\end{abstract}

\section{Introduction}

In this paper, we derive necessary and sufficient conditions for the existence of relative extrema for analytic functions on a closed interval $[a, b]$ of the Levi-Civita field $\mathcal{R}$. Moreover, the extreme value theorem, the mean value theorem and the inverse function theorem will be shown to hold for such functions. We recall that the elements of $\mathcal{R}$ and its complex counterpart $\mathcal{C}$ are functions from $\mathbb{Q}$ to $\mathbb{R}$ and $\mathbb{C}$, respectively, with left-finite support (denoted by supp). That is, below every rational number $q$, there are only finitely many points where the given function does not vanish. For the further discussion, it is convenient to introduce the following terminology.

2000 Mathematics Subject Classification. 11D88, 12J25, 26E30, 30 G06.

Key words and phrases. Levi-Civita field, non-Archimedean analysis, power series, analytic functions, extreme value theorem, mean value theorem.

(C)2011 American Mathematical Society 
Definition 1.1. $(\lambda, \sim, \approx)$ For $x \neq 0$ in $\mathcal{R}$ or $\mathcal{C}$, we let $\lambda(x)=\min (\operatorname{supp}(x))$, which exists because of the left-finiteness of $\operatorname{supp}(x)$; and we let $\lambda(0)=+\infty$.

Given $x, y \neq 0$ in $\mathcal{R}$ or $\mathcal{C}$, we say $x \sim y$ if $\lambda(x)=\lambda(y)$; and we say $x \approx y$ if $\lambda(x)=\lambda(y)$ and $x[\lambda(x)]=y[\lambda(y)]$.

At this point, these definitions may feel somewhat arbitrary; but after having introduced an order on $\mathcal{R}$, we will see that $\lambda$ describes orders of magnitude, the relation $\approx$ corresponds to agreement up to infinitely small relative error, while $\sim$ corresponds to agreement of order of magnitude.

The sets $\mathcal{R}$ and $\mathcal{C}$ are endowed with formal power series multiplication and componentwise addition, which make them into fields $[\mathbf{3}]$ in which we can isomorphically embed $\mathbb{R}$ and $\mathbb{C}$ (respectively) as subfields via the map $\Pi: \mathbb{R}, \mathbb{C} \rightarrow \mathcal{R}, \mathcal{C}$ defined by

$$
\Pi(x)[q]=\left\{\begin{array}{ll}
x & \text { if } q=0 \\
0 & \text { else }
\end{array} .\right.
$$

Definition 1.2. (Order in $\mathcal{R}$ ) Let $x \neq y$ in $\mathcal{R}$ be given. Then we say $x>y$ if $(x-y)[\lambda(x-y)]>0$; furthermore, we say $x<y$ if $y>x$.

With this definition of the order relation, $\mathcal{R}$ is an ordered field. Moreover, the embedding $\Pi$ in Equation (1.1) of $\mathbb{R}$ into $\mathcal{R}$ is compatible with the order. The order induces an absolute value on $\mathcal{R}$ in the natural way; and an absolute value on $\mathcal{C}$ is obtained in the same way as in $\mathbb{C}:|x+i y|=\sqrt{x^{2}+y^{2}}$. We also note here that $\lambda$, as defined above, is a valuation; moreover, the relation $\sim$ is an equivalence relation, and the set of equivalence classes (the value group) is (isomorphic to) $\mathbb{Q}$.

Besides the usual order relations, some other notations are convenient.

Definition 1.3. ( $\ll, \gg)$ Let $x, y \in \mathcal{R}$ be non-negative. We say $x$ is infinitely smaller than $y$ (and write $x \ll y$ ) if $n x<y$ for all $n \in \mathbb{N}$; we say $x$ is infinitely larger than $y$ (and write $x \gg y$ ) if $y \ll x$. If $x \ll 1$, we say $x$ is infinitely small; if $x \gg 1$, we say $x$ is infinitely large. Infinitely small numbers are also called infinitesimals or differentials. Infinitely large numbers are also called infinite. Non-negative numbers that are neither infinitely small nor infinitely large are also called finite.

Definition 1.4. (The Number $d$ ) Let $d$ be the element of $\mathcal{R}$ given by $d[1]=1$ and $d[q]=0$ for $q \neq 1$.

It is easy to check that $d^{q} \ll 1$ if $q>0$ and $d^{q} \gg 1$ if $q<0$. Moreover, for all $x \in \mathcal{R}(\operatorname{resp} . \mathcal{C})$, the elements of $\operatorname{supp}(x)$ can be arranged in ascending order, say $\operatorname{supp}(x)=\left\{q_{1}, q_{2}, \ldots\right\}$ with $q_{j}<q_{j+1}$ for all $j$; and $x$ can be written as $x=\sum_{j=1}^{\infty} x\left[q_{j}\right] d^{q_{j}}$, where the series converges in the topology induced by the absolute value $[\mathbf{3}]$.

Altogether, it follows that $\mathcal{R}$ is a non-Archimedean field extension of $\mathbb{R}$. For a detailed study of this field, we refer the reader to $[3,14,15,21,16,17,22$, $18,19,20,13]$. In particular, it is shown that $\mathcal{R}$ is complete with respect to the topology induced by the absolute value. In the wider context of valuation theory, it is interesting to note that the topology induced by the absolute value, the so-called strong topology, is the same as that introduced via the valuation $\lambda$, as was shown in $[\mathbf{1 8}]$.

It follows therefore that the fields $\mathcal{R}$ and $\mathcal{C}$ are just special cases of the class of fields discussed in [11]. For a general overview of the algebraic properties of formal 
power series fields in general, we refer the reader to the comprehensive overview by Ribenboim [10], and for an overview of the related valuation theory to the books by Krull [4], Schikhof [11] and Alling [1]. A thorough and complete treatment of ordered structures can also be found in [9].

Besides being the smallest ordered non-Archimedean field extension of the real numbers that is both complete in the order topology and real closed, the LeviCivita field $\mathcal{R}$ is of particular interest because of its practical usefulness. Since the supports of the elements of $\mathcal{R}$ are left-finite, it is possible to represent these numbers on a computer [3]. Having infinitely small numbers, the errors in classical numerical methods can be made infinitely small and hence irrelevant in all practical applications. One such application is the computation of derivatives of real functions representable on a computer [14], where both the accuracy of formula manipulators and the speed of classical numerical methods are achieved.

In $[\mathbf{1 5}, \mathbf{1 8}, \mathbf{2 0}]$, we study the convergence and analytical properties of power series in a topology weaker than the valuation topology used in [11], and thus allow for a much larger class of power series to be included in the study. Previous work on power series on the Levi-Civita fields $\mathcal{R}$ and $\mathcal{C}$ had been mostly restricted to power series with real or complex coefficients. In $[\mathbf{6}, \mathbf{7}, \mathbf{8}, \mathbf{5}]$, they could be studied for infinitely small arguments only, while in $[\mathbf{3}]$, using the newly introduced weak topology, also finite arguments were possible. Moreover, power series over complete valued fields in general have been studied by Schikhof [11], Alling [1] and others in valuation theory, but always in the valuation topology.

In [15], we study the general case when the coefficients in the power series are Levi-Civita numbers, using the weak convergence of [3]. We derive convergence criteria for power series which allow us to define a radius of convergence $\eta$ such that the power series converges weakly for all points whose distance from the center is smaller than $\eta$ by a finite amount and it converges strongly for all points whose distance from the center is infinitely smaller than $\eta$. Then, in [18], we study the analytical properties of power series on $\mathcal{R}$ and $\mathcal{C}$ within their domain of convergence. We show that, within their radius of convergence, power series are infinitely often differentiable and the derivatives to any order are obtained by differentiating the power series term by term. Also, power series can be re-expanded around any point in their domain of convergence and the radius of convergence of the new series is equal to the difference between the radius of convergence of the original series and the distance between the original and new centers of the series. We then study a class of functions that are given locally by power series (which we call $\mathcal{R}$-analytic functions) and show that they are closed under arithmetic operations and compositions and they are infinitely often differentiable with the derivative functions of all orders being $\mathcal{R}$-analytic themselves.

In $[\mathbf{2 0}]$, we focus on the proof of the intermediate value theorem for the $\mathcal{R}$ analytic functions. Given a function $f$ that is $\mathcal{R}$-analytic on an interval $[a, b]$ and a value $S$ between $f(a)$ and $f(b)$, we use iteration to construct a sequence of numbers in $[a, b]$ that converges strongly to a point $c \in[a, b]$ such that $f(c)=S$. The proof is quite involved, making use of many of the results proved in $[\mathbf{1 5}, \mathbf{1 8}]$ as well as some results from Real Analysis.

This paper is a continuation of the study of power series and $\mathcal{R}$-analytic functions in $[\mathbf{1 5}, \mathbf{1 8}, \mathbf{2 0}]$. First we state and prove necessary and sufficient conditions for the existence of relative extrema. Then we use that as well as the intermediate 
value theorem and its proof to prove the extreme value theorem, the mean value theorem, and the inverse function theorem for functions that are $\mathcal{R}$-analytic on an interval $[a, b]$, thus showing that such functions behave as nicely as real analytic functions.

\section{Review of Power Series and $\mathcal{R}$-Analytic Functions}

We start this section with a brief review of the convergence of sequences in two different topologies; and we refer the reader to [15] for a more detailed study.

Definition 2.1. A sequence $\left(s_{n}\right)$ in $\mathcal{R}$ or $\mathcal{C}$ is called regular if the union of the supports of all members of the sequence is a left-finite subset of $\mathbb{Q}$. (Recall that $A \subset \mathbb{Q}$ is said to be left-finite if for every $q \in \mathbb{Q}$ there are only finitely many elements in $A$ that are smaller than q.)

Definition 2.2. We say that a sequence $\left(s_{n}\right)$ converges strongly in $\mathcal{R}$ or $\mathcal{C}$ if it converges in the order (valuation) topology.

It is shown in [2] that the fields $\mathcal{R}$ and $\mathcal{C}$ are complete with respect to the order topology; and a detailed study of strong convergence can be found in $[\mathbf{1 2}, \mathbf{1 5}]$.

Since power series with real (complex) coefficients do not converge strongly for any nonzero real (complex) argument, it is advantageous to study a new kind of convergence. We do that by defining a family of semi-norms on $\mathcal{R}$ or $\mathcal{C}$, which induces a topology weaker than the topology induced by the absolute value and called weak topology $[3,12,15,13]$.

Definition 2.3. Given $r \in \mathbb{R}$, we define a mapping $\|\cdot\|_{r}: \mathcal{R}$ or $\mathcal{C} \rightarrow \mathbb{R}$ as follows: $\|x\|_{r}=\max \{|x[q]|: q \in \mathbb{Q}$ and $q \leq r\}$.

The maximum in Definition 2.3 exists in $\mathbb{R}$ since, for any $r \in \mathbb{R}$, only finitely many of the $x[q]$ 's considered do not vanish.

Definition 2.4. A sequence $\left(s_{n}\right)$ in $\mathcal{R}$ (resp. $\mathcal{C}$ ) is said to be weakly convergent if there exists $s \in \mathcal{R}$ (resp. $\mathcal{C}$ ), called the weak limit of the sequence $\left(s_{n}\right)$, such that for all $\epsilon>0$ in $\mathbb{R}$, there exists $N \in \mathbb{N}$ such that $\left\|s_{m}-s\right\|_{1 / \epsilon}<\epsilon$ for all $m \geq N$.

It is shown $[\mathbf{3}]$ that $\mathcal{R}$ and $\mathcal{C}$ are not Cauchy complete with respect to the weak topology and that strong convergence implies weak convergence to the same limit. A detailed study of weak convergence is found in $[\mathbf{3}, \mathbf{1 2}, \mathbf{1 5}]$.

2.1. Power Series. In the following, we review strong and weak convergence criteria for power series, Theorem 2.5 and Theorem 2.6, the proofs of which are given in [15]. We also note that, since strong convergence is equivalent to convergence with respect to the valuation topology, Theorem 2.5 is a special case of the result on page 59 of $[\mathbf{1 1}]$.

Theorem 2.5. (Strong Convergence Criterion for Power Series) Let $\left(a_{n}\right)$ be a sequence in $\mathcal{R}$ (resp. $\mathcal{C}$ ), and let

$$
\lambda_{0}=\limsup _{n \rightarrow \infty}\left(\frac{-\lambda\left(a_{n}\right)}{n}\right) \text { in } \mathbb{R} \cup\{-\infty, \infty\} .
$$

Let $x_{0} \in \mathcal{R}$ (resp. $\mathcal{C}$ ) be fixed and let $x \in \mathcal{R}$ (resp. $\mathcal{C}$ ) be given. Then the power series $\sum_{n=0}^{\infty} a_{n}\left(x-x_{0}\right)^{n}$ converges strongly if $\lambda\left(x-x_{0}\right)>\lambda_{0}$ and is strongly divergent if $\lambda\left(x-x_{0}\right)<\lambda_{0}$ or if $\lambda\left(x-x_{0}\right)=\lambda_{0}$ and $-\lambda\left(a_{n}\right) / n>\lambda_{0}$ for infinitely many $n$. 
Theorem 2.6. (Weak Convergence Criterion for Power Series) Let $\left(a_{n}\right)$ be a sequence in $\mathcal{R}($ resp. $\mathcal{C})$, and let $\lambda_{0}=\limsup _{n \rightarrow \infty}\left(-\lambda\left(a_{n}\right) / n\right) \in \mathbb{Q}$. Let $x_{0} \in \mathcal{R}$ (resp. $\mathcal{C}$ ) be fixed, and let $x \in \mathcal{R}$ (resp. $\mathcal{C}$ ) be such that $\lambda\left(x-x_{0}\right)=\lambda_{0}$. For each $n \geq 0$, let $b_{n}=a_{n} d^{n \lambda_{0}}$. Suppose that the sequence $\left(b_{n}\right)$ is regular and write $\bigcup_{n=0}^{\infty} \operatorname{supp}\left(b_{n}\right)=\left\{q_{1}, q_{2}, \ldots\right\}$; with $q_{j_{1}}<q_{j_{2}}$ if $j_{1}<j_{2}$. For each $n$, write $b_{n}=$ $\sum_{j=1}^{\infty} b_{n_{j}} d^{q_{j}}$, where $b_{n_{j}}=b_{n}\left[q_{j}\right]$. Let

$$
\eta=\frac{1}{\sup \left\{\lim \sup _{n \rightarrow \infty}\left|b_{n_{j}}\right|^{1 / n}: j \geq 1\right\}} \text { in } \mathbb{R} \cup\{\infty\},
$$

with the conventions $1 / 0=\infty$ and $1 / \infty=0$. Then $\sum_{n=0}^{\infty} a_{n}\left(x-x_{0}\right)^{n}$ converges absolutely weakly if $\left|\left(x-x_{0}\right)\left[\lambda_{0}\right]\right|<\eta$ and is weakly divergent if $\left|\left(x-x_{0}\right)\left[\lambda_{0}\right]\right|>\eta$.

Remark 2.7. The number $\eta$ in Equation (2.1) will be referred to as the radius of weak convergence of the power series $\sum_{n=0}^{\infty} a_{n}\left(x-x_{0}\right)^{n}$.

As an immediate consequence of Theorem 2.6, we obtain the following result which allows us to extend real and complex functions representable by power series to the Levi-Civita fields $\mathcal{R}$ and $\mathcal{C}$.

Corollary 2.8. (Power Series with Purely Real or Complex Coefficients) Let $\sum_{n=0}^{\infty} a_{n} X^{n}$ be a power series with purely real (resp. complex) coefficients and with classical radius of convergence equal to $\eta$. Let $x \in \mathcal{R}$ (resp. $\mathcal{C}$ ), and let $A_{n}(x)=\sum_{j=0}^{n} a_{j} x^{j} \in \mathcal{R}$ (resp. $\mathcal{C}$ ). Then, for $|x|<\eta$ and $|x| \not \approx \eta$, the sequence $\left(A_{n}(x)\right)$ converges absolutely weakly. We define the limit to be the continuation of the power series to $\mathcal{R}$ (resp. $\mathcal{C}$ ).

2.2. $\mathcal{R}$-Analytic Functions. In this section, we review the algebraic and analytical properties of a class of functions that are given locally by power series and we refer the reader to $[\mathbf{1 8}, \mathbf{2 0}]$ for a more detailed study.

Definition 2.9. Let $a<b$ in $\mathcal{R}$ be given and let $f:[a, b] \rightarrow \mathcal{R}$. Then we say that $f$ is expandable or $\mathcal{R}$-analytic on $[a, b]$ if for all $x \in[a, b]$ there exists a positive $\delta \sim b-a$ in $\mathcal{R}$, and there exists a regular sequence $\left(a_{n}(x)\right)$ in $\mathcal{R}$ such that, under weak convergence, $f(y)=\sum_{n=0}^{\infty} a_{n}(x)(y-x)^{n}$ for all $y \in(x-\delta, x+\delta) \cap[a, b]$.

It is shown in [18] that if $f$ is $\mathcal{R}$-analytic on $[a, b]$ then $f$ is bounded on $[a, b]$; also, if $g$ is $\mathcal{R}$-analytic on $[a, b]$ and $\alpha \in \mathcal{R}$ then $f+\alpha g$ and $f \cdot g$ are $\mathcal{R}$-analytic on $[a, b]$. Moreover, the composition of $\mathcal{R}$-analytic functions is $\mathcal{R}$-analytic. Furthermore, using the fact that power series on $\mathcal{R}$ are infinitely often differentiable within their domain of convergence and the derivatives to any order are obtained by differentiating the power series term by term [18], we obtain the following result.

THEOREM 2.10. Let $a<b$ in $\mathcal{R}$ be given, and let $f:[a, b] \rightarrow \mathcal{R}$ be $\mathcal{R}$-analytic on $[a, b]$. Then $f$ is infinitely often differentiable on $[a, b]$, and for any positive integer $m$, we have that $f^{(m)}$ is $\mathcal{R}$-analytic on $[a, b]$. Moreover, if $f$ is given locally around $x_{0} \in[a, b]$ by $f(x)=\sum_{n=0}^{\infty} a_{n}\left(x_{0}\right)\left(x-x_{0}\right)^{n}$, then $f^{(m)}$ is given by

$$
f^{(m)}(x)=\sum_{n=m}^{\infty} n(n-1) \cdots(n-m+1) a_{n}\left(x_{0}\right)\left(x-x_{0}\right)^{n-m} .
$$

In particular, we have that $a_{m}\left(x_{0}\right)=f^{(m)}\left(x_{0}\right) / m$ ! for all $m=0,1,2, \ldots$ 
In $[\mathbf{2 0}]$, we prove the intermediate value theorem for $\mathcal{R}$-analytic functions on an interval $[a, b]$. A close look at that proof shows that if $f$ is not constant on $[a, b]$ and $S$ is between $f(a)$ and $f(b)$ then there are only finitely many points $c$ in $[a, b]$ such that $f(c)=S$. This will be crucial for the proof of the extreme value theorem for the $\mathcal{R}$-analytic functions in the next section.

\section{Relative and Absolute Extrema, the Mean Value Theorem, and the Inverse Function Theorem for $\mathcal{R}$-Analytic Functions}

In this section, we complete the study of $\mathcal{R}$-analytic functions which was started in $[\mathbf{1 8}, \mathbf{2 0}]$ by proving the extreme value theorem, the mean value theorem and the inverse function theorem for these functions and thus showing that $\mathcal{R}$-analytic functions have all the nice properties of real analytic functions.

TheOREm 3.1. Let $a<b$ in $\mathcal{R}$ be given; let $f:[a, b] \rightarrow \mathcal{R}$ be $\mathcal{R}$-analytic on $[a, b]$; let $x_{0} \in(a, b)$ and let $m \in \mathbb{N}$ be the order of the first nonvanishing derivative of $f$ at $x_{0}$. Then $f$ has a relative extremum at $x_{0}$ if and only if $m$ is even. In that case ( $m$ is even), the extremum is a minimum if $f^{(m)}\left(x_{0}\right)>0$ and a maximum if $f^{(m)}\left(x_{0}\right)<0$.

Proof. Since $f$ is $\mathcal{R}$-analytic on $[a, b]$, there exists $\delta_{0}>0$ in $\mathcal{R}$ such that, for all $h \in\left(-\delta_{0}, \delta_{0}\right)$, we have that

$$
f\left(x_{0}+h\right)=f\left(x_{0}\right)+\frac{f^{(m)}\left(x_{0}\right)}{m !} h^{m}+\sum_{j=m+1}^{\infty} \frac{f^{(j)}\left(x_{0}\right)}{j !} h^{j} .
$$

Since the sequence $\left(f^{(j)}\left(x_{0}\right)\right)$ is regular, it is uniformly bounded; thus there exists $M>0$ in $\mathcal{R}$ such that

$$
\left|f^{(j)}\left(x_{0}\right)\right|<M, \text { for all } j \geq m
$$

Let

$$
\delta=\min \left\{\delta_{0}, d, d \frac{\left|f^{(m)}\left(x_{0}\right)\right|}{M}\right\} .
$$

Then, for all $h \in(-\delta, \delta)$ and for all $j \geq m+1$, we have that

$$
\left|f^{(j)}\left(x_{0}\right)\right||h|^{j-m}<M \delta^{j-m} \leq M \delta \leq d\left|f^{(m)}\left(x_{0}\right)\right| \ll\left|f^{(m)}\left(x_{0}\right)\right| .
$$

It follows that

$$
\left|f^{(j)}\left(x_{0}\right)\right||h|^{j} \ll\left|f^{(m)}\left(x_{0}\right)\right||h|^{m} \text { for all } j \geq m+1 \text {; }
$$

and hence

$$
f\left(x_{0}+h\right)-f\left(x_{0}\right) \approx \frac{f^{(m)}\left(x_{0}\right)}{m !} h^{m} \text { for all } h \in(-\delta, \delta) .
$$

It then readily follows that

$f$ has a relative maximum (minimum) at $x_{0}$

$\Leftrightarrow f\left(x_{0}+h\right)-f\left(x_{0}\right)<0$ (resp. $\left.f\left(x_{0}+h\right)-f\left(x_{0}\right)>0\right)$ for $|h|$ small enough

$\Leftrightarrow m$ is even and $f^{(m)}\left(x_{0}\right)<0$ (resp. $f^{(m)}\left(x_{0}\right)>0$ ), using Equation (3.1). 
Corollary 3.2. Let $f:[a, b] \rightarrow \mathcal{R}$ be $\mathcal{R}$-analytic on $[a, b]$; and let $x_{0} \in(a, b)$ be given. Suppose there exists $\Delta>0$ in $\mathcal{R}$ such that $f^{\prime}(x)>0$ for all $x \in\left(x_{0}-\Delta, x_{0}\right)$ and $f^{\prime}(x)<0$ for all $x \in\left(x_{0}, x_{0}+\Delta\right)$ (resp. $f^{\prime}(x)<0$ for all $x \in\left(x_{0}-\Delta, x_{0}\right)$ and $f^{\prime}(x)>0$ for all $x \in\left(x_{0}, x_{0}+\Delta\right)$ ). Then $f$ has a relative maximum (resp. minimum) at $x_{0}$ and $f^{\prime}\left(x_{0}\right)=0$.

Proof. Let $m$ be the order of the first nonvanishing derivative of $f$ at $x_{0}$; and let $\delta<\Delta$ in $\mathcal{R}$ be as in the proof of Theorem 3.1 above. Then, for all $h \in(-\delta, \delta)$, we have that

$$
\begin{aligned}
f\left(x_{0}+h\right) & =f\left(x_{0}\right)+\frac{f^{(m)}\left(x_{0}\right)}{m !} h^{m}+\sum_{j=m+1}^{\infty} \frac{f^{(j)}\left(x_{0}\right)}{j !} h^{j} ; \\
f\left(x_{0}+h\right)-f\left(x_{0}\right) & \approx \frac{f^{(m)}\left(x_{0}\right)}{m !} h^{m} ; \\
f^{\prime}\left(x_{0}+h\right) & =\frac{f^{(m)}\left(x_{0}\right)}{(m-1) !} h^{m-1}+\sum_{j=m+1}^{\infty} \frac{f^{(j)}\left(x_{0}\right)}{(j-1) !} h^{j-1} ; \\
f^{\prime}\left(x_{0}+h\right) & \approx \frac{f^{(m)}\left(x_{0}\right)}{(m-1) !} h^{m-1} .
\end{aligned}
$$

It follows from the last equation above and the hypothesis of the corollary that $m-1$ is odd and hence $m$ is even. Also, $f^{(m)}\left(x_{0}\right)<0$ (resp. $f^{(m)}\left(x_{0}\right)>0$ ). Thus, $f$ has a relative maximum (resp. minimum) at $x_{0}$.

Finally, since $m$ is even, we infer that $f^{\prime}\left(x_{0}\right)=0$. The same conclusion also follows from applying the intermediate value theorem to $f^{\prime}$ which is $\mathcal{R}$-analytic on $[a, b]$ and hence on $[-\delta, \delta]$.

TheOREM 3.3. (Extreme Value Theorem) Let $a<b$ in $\mathcal{R}$ be given and let $f:[a, b] \rightarrow \mathcal{R}$ be $\mathcal{R}$-analytic on $[a, b]$. Then $f$ assumes a maximum and a minimum on $[a, b]$.

Proof. First we show that $f$ assumes a maximum on $[a, b]$. Without loss of generality we may assume that $f$ is not constant on $[a, b]$. Let $F:[0,1] \rightarrow \mathcal{R}$ be given by

$$
F(x)=f((b-a) x+a) .
$$

Then $F$ is $\mathcal{R}$-analytic on [0,1]; and $f$ assumes a maximum on $[a, b]$ if and only if $F$ assumes a maximum on $[0,1]$. So without loss of generality, we may assume that $a=0, b=1$, and $f=F$. Also, since scaling the function by a positive constant factor does not affect the existence of an absolute maximum, we may assume that $f$ has a zero index on $[a, b]=[0,1]$; that is,

$$
i(f):=\min \{\operatorname{supp}(f(x)): x \in[0,1]\}=0 .
$$

That the minimum in Equation (3.2) exists was shown in [18] (Remark 4.8 and Corollary 4.9).

Let $f_{R}:[0,1] \cap \mathbb{R} \rightarrow \mathbb{R}$ be given by $f_{R}(X)=f(X)[0]$. Then $f_{R}$ is not constant and $\mathbb{R}$-analytic on $[0,1] \cap \mathbb{R}$, as we will show next. Let $X \in[0,1] \cap \mathbb{R}$ be given. Then, by Definition 2.9 and Theorem 2.10, there is a finite $\delta>0$ (which without loss of generality we may take to be real) such that

$$
f(X+h)=f(X)+\sum_{n=1}^{\infty} \frac{f^{(n)}(X)}{n !} h^{n}
$$


for all $h \in \mathcal{R}$ satisfying $|h|<\delta$ and $X+h \in[0,1]$. In particular, it follows that

$$
f(X+h)=f(X)+\sum_{n=1}^{\infty} \frac{f^{(n)}(X)}{n !} h^{n}
$$

for all $h \in \mathbb{R}$ satisfying $|h|<\delta$ and $X+h \in[0,1] \cap \mathbb{R}$. Taking the real parts of both sides of the equation above, we get:

$$
f_{R}(X+h)=f_{R}(X)+\sum_{n=1}^{\infty} \frac{f^{(n)}(X)[0]}{n !} h^{n} .
$$

That the real power series on the right side of Equation (3.4) converges in the order topology of $\mathbb{R}$ to $f_{R}(X+h)$ follows from Theorem 2.13 in [15]. Thus, we have shown that for all $X \in[0,1] \cap \mathbb{R}$ there is a real $\delta>0$ such that

$$
f_{R}(X+h)=f_{R}(X)+\sum_{n=1}^{\infty} \frac{f^{(n)}(X)[0]}{n !} h^{n}
$$

for all $h \in \mathbb{R}$ satisfying $|h|<\delta$ and $X+h \in[0,1] \cap \mathbb{R}$. This shows that $f_{R}$ is $\mathbb{R}$-analytic on $[0,1] \cap \mathbb{R}$; in particular, $f_{R}$ is a continuous and infinitely often differentiable real-valued function on the real interval $[0,1] \cap \mathbb{R}$, with

$$
f_{R}^{(n)}(X)=f^{(n)}(X)[0] \text { for all } X \in[0,1] \cap \mathbb{R} .
$$

Since $f_{R}$ is continuous on $[0,1] \cap \mathbb{R}, f_{R}$ assumes a maximum $M_{R}$ on $[0,1] \cap \mathbb{R}$; and since $f_{R}$ is $\mathbb{R}$-analytic on $[0,1] \cap \mathbb{R}$, there are only finitely many points $X_{1}, \ldots, X_{k}$ in $[0,1] \cap \mathbb{R}$ where $f_{R}$ assumes its maximum $M_{R}$.

We look for a maximum of $f$ in the infinitely small neighborhoods of the $X_{j}$ 's. So let $j \in\{1, \ldots, k\}$ be given. Assume $X_{j} \in(0,1)$. Then $f_{R}^{\prime}\left(X_{j}\right)=0$ and there exists a real $\delta_{1}>0$ such that $f_{R}^{\prime}(X)>0$ for $X \in\left(X_{j}-\delta_{1}, X_{j}\right) \cap \mathbb{R}$ and $f_{R}^{\prime}(X)<0$ for $X \in\left(X_{j}, X_{j}+\delta_{1}\right) \cap \mathbb{R}$. Since $f$ is $\mathcal{R}$-analytic on $[0,1]$, there exists a real $\delta \leq \delta_{1}$ such that

$$
f\left(X_{j}+h\right)=\sum_{n=0}^{\infty} \frac{f^{(n)}\left(X_{j}\right)}{n !} h^{n} \text { and } f^{\prime}\left(X_{j}+h\right)=\sum_{n=1}^{\infty} \frac{f^{(n)}\left(X_{j}\right)}{(n-1) !} h^{n-1}
$$

for $0 \leq|h|<\delta$. Let $m=\min \left\{n \in \mathbb{N}: \lambda\left(f^{(n+1)}\left(X_{j}\right)\right)=0\right\}$. Note that the condition $\lambda\left(f^{(m+1)}\left(X_{j}\right)\right)=0$ is equivalent to $f_{R}^{(m+1)}\left(X_{j}\right) \neq 0$. Thus, $m+1$ is the order of the first nonvanishing derivative of the real function $f_{R}$ at the real point $X_{j} \in(0,1)$. Since $f_{R}$ assumes its absolute maximum at $X_{j}, f_{R}$ has a relative maximum at $X_{j}$ and hence $m+1$ is even; i.e. $m$ is odd.

Using the intermediate value theorem and its proof $[\mathbf{2 0}]$, applied to $f^{\prime}$, we obtain at least one and at most $m$ roots of $f^{\prime}$ that are infinitely close to $X_{j}$, and $f^{\prime}$ changes sign from positive to negative in going from the left to the right of at least one of the roots. Thus we obtain at least one relative extremum (one relative maximum with no relative minima) and at most $m$ relative extrema $\left(\frac{m+1}{2}\right.$ relative maxima and $\frac{m-1}{2}$ relative minima) of $f$ in the infinitely small neighborhood of $X_{j}$. Since $f$ satisfies the intermediate value theorem on any subinterval of $[0,1]$, it follows that the largest of the finitely many relative maximum values attained by $f$ in the infinitely small neighborhood of $X_{j}$ is the absolute maximum value in that neighborhood; let

$$
M_{j}=\max \left\{f\left(X_{j}+h\right):|h| \ll 1\right\} .
$$


Similarly we show that $f$ has a maximum in the infinitely small neighborhood of $a=0$ if $0 \in\left\{X_{1}, \ldots, X_{k}\right\}$ and that $f$ has a maximum in the infinitely small neighborhood of $b=1$ if $1 \in\left\{X_{1}, \ldots, X_{k}\right\}$.

Let

$$
M=\max \left\{M_{j}: 1 \leq j \leq k\right\} .
$$

We show that $M=\max \{f(x): x \in[0,1]\}$. So let $x \in[0,1]$ be given. Suppose $x$ is finitely away from $X_{j}$ for all $j \in\{1, \ldots, k\}$. Then

$$
f(x)-M=\left(f(x)-f_{R}(x[0])\right)+\left(f_{R}(x[0])-M_{R}\right)+\left(M_{R}-M\right) .
$$

Since $x[0] \notin\left\{X_{1}, \ldots, X_{k}\right\}$, we have that $f_{R}(x[0])-M_{R}$ is negative and finite in absolute value. Since $\left|f(x)-f_{R}(x[0])\right| \ll 1$ and since $\left|M_{R}-M\right| \ll 1$, we obtain that $f(x)-M<0$; that is $f(x)<M$. Now suppose $x$ is infinitely close to one of the $X_{j}$ 's, say $X_{j_{0}}$. Then $f(x) \leq M_{j_{0}} \leq M$. Hence $f(x) \leq M$ for all $x \in[0,1]$. Moreover, $M$ is assumed on $[0,1]$ since each of the $M_{j}$ 's in Equation (3.5) is. Thus $M=\max \{f(x): x \in[0,1]\}$.

Applying the above arguments to $-f$, we infer that $-f$ assumes a maximum on $[a, b]$. Thus, $f$ assumes also a minimum on $[a, b]$.

Altogether, as a consequence of the intermediate value theorem and the extreme value theorem, we obtain the following result.

Corollary 3.4. Let $a<b$ in $\mathcal{R}$ be given and let $f:[a, b] \rightarrow \mathcal{R}$ be $\mathcal{R}$-analytic on $[a, b]$. Then there exist $m, M \in \mathcal{R}$ such that $f([a, b])=[m, M]$.

Corollary 3.5. (Rolle's Theorem) Let $a<b$ in $\mathcal{R}$ be given and let $f:[a, b] \rightarrow$ $\mathcal{R}$ be $\mathcal{R}$-analytic. Suppose $f(a)=f(b)$. Then there exists $c \in(a, b)$ such that $f^{\prime}(c)=0$.

Proof. If $f(x)=f(a)$ for all $x \in[a, b]$, then $f^{\prime}(x)=0$ for all $x \in(a, b)$ and we are done. So we may assume that $f$ is not constant on $[a, b]$. Then $f$ assumes its (absolute) maximum or minimum at some $c \in(a, b)$. It follows that $f$ has a relative extremum at $c$; and hence $f^{\prime}(c)=0$, by Theorem 3.1 .

Corollary 3.6. (Mean Value Theorem) Let $a<b$ in $\mathcal{R}$ be given and let $f:[a, b] \rightarrow \mathcal{R}$ be $\mathcal{R}$-analytic on $[a, b]$. Then there exists $c \in(a, b)$ such that

$$
f^{\prime}(c)=\frac{f(b)-f(a)}{b-a} .
$$

Proof. Let $h:[a, b] \rightarrow \mathcal{R}$ be given by

$$
h(x)=f(x)-f(a)-\frac{f(b)-f(a)}{b-a}(x-a) .
$$

Then $h$ is $\mathcal{R}$-analytic on $[a, b]$. Moreover, $h(a)=h(b)=0$. Thus, by Rolle's theorem (Corollary 3.5), there exists $c \in(a, b)$ such that

$$
0=h^{\prime}(c)=f^{\prime}(c)-\frac{f(b)-f(a)}{b-a} ; \text { and hence } f^{\prime}(c)=\frac{f(b)-f(a)}{b-a} .
$$

Corollary 3.7. Let $a<b$ in $\mathcal{R}$ be given, and let $f:[a, b] \rightarrow \mathcal{R}$ be $\mathcal{R}$-analytic on $[a, b]$. Then the following are true. 
(i) If $f^{\prime}(x) \neq 0$ for all $x \in(a, b)$ then either $f^{\prime}(x)>0$ for all $x \in(a, b)$ and $f$ is strictly increasing on $[a, b]$, or $f^{\prime}(x)<0$ for all $x \in(a, b)$ and $f$ is strictly decreasing on $[a, b]$.

(ii) If $f^{\prime}(x)=0$ for all $x \in(a, b)$, then $f$ is constant on $[a, b]$.

Proof. Suppose $f^{\prime}(x) \neq 0$ for all $x \in(a, b)$. Then applying the intermediate value theorem to $f^{\prime}$, we infer that either $f^{\prime}(x)>0$ for all $x \in(a, b)$ or $f^{\prime}(x)<0$ for all $x \in(a, b)$. First assume that $f^{\prime}(x)>0$ for all $x \in(a, b)$ and let $x, y \in[a, b]$ be such that $y>x$. By Corollary 3.6, there exists $c \in(x, y) \subset(a, b)$ such that

$$
f^{\prime}(c)=\frac{f(y)-f(x)}{y-x} .
$$

Since $c \in(a, b)$, we have that $f^{\prime}(c)>0$; and hence $f(y)>f(x)$. Thus, $f$ is strictly increasing on $[a, b]$. Similarly, we can show that $f$ is strictly decreasing on $[a, b]$ if $f^{\prime}(x)<0$ for all $x \in(a, b)$.

Now suppose $f^{\prime}(x)=0$ for all $x \in(a, b)$, and let $y \in(a, b]$ be given. Then there exists $c \in(a, y) \subset(a, b)$ such that

$$
f^{\prime}(c)=\frac{f(y)-f(a)}{y-a} .
$$

Since $c \in(a, b)$, we have that $f^{\prime}(c)=0$; and hence $f(y)=f(a)$. This shows that $f$ is constant on $[a, b]$.

COROLlaRY 3.8. (Inverse Function Theorem) Let $a<b$ in $\mathcal{R}$ be given, let $f:[a, b] \rightarrow \mathcal{R}$ be $\mathcal{R}$-analytic on $[a, b]$, and let $x_{0} \in(a, b)$ be such that $f^{\prime}\left(x_{0}\right)>0$ (resp. $\left.f^{\prime}\left(x_{0}\right)<0\right)$. Then there exists $\delta>0$ in $\mathcal{R}$ such that

(i) $f^{\prime}>0$ and $f$ is strictly increasing (resp. $f^{\prime}<0$ and $f$ is strictly decreasing) on $\left[x_{0}-\delta, x_{0}+\delta\right]$.

(ii) $f\left(\left[x_{0}-\delta, x_{0}+\delta\right]\right)=[m, M]$ where $m=f\left(x_{0}-\delta\right)$ and $M=f\left(x_{0}+\delta\right)$ (resp. $m=f\left(x_{0}+\delta\right)$ and $M=f\left(x_{0}-\delta\right)$ ).

(iii) $\exists g:[m, M] \rightarrow\left[x_{0}-\delta, x_{0}+\delta\right]$, strictly increasing (resp. strictly decreasing) on $[m, M]$, such that

$-g$ is the inverse of $f$ on $\left[x_{0}-\delta, x_{0}+\delta\right]$;

- $g$ is differentiable on $[m, M]$; and for all $y \in[m, M]$,

$$
g^{\prime}(y)=\frac{1}{f^{\prime}(g(y))} .
$$

Proof. Assume first that $f^{\prime}\left(x_{0}\right)>0$.

(i) By continuity of $f^{\prime}$ at $x_{0} \in(a, b)$, there exists $\delta>0$ in $\mathcal{R}$ such that $\left(x_{0}-\right.$ $\left.\delta, x_{0}+\delta\right) \subset(a, b)$ and $f^{\prime}(x)>0$ for all $x \in\left(x_{0}-\delta, x_{0}+\delta\right)$. Since $\left[x_{0}-\delta, x_{0}+\delta\right] \subset[a, b]$, it follows that $f$ is $\mathcal{R}$-analytic on $\left[x_{0}-\delta, x_{0}+\delta\right]$. Then by Corollary $3.7, f$ is strictly increasing on $\left[x_{0}-\delta, x_{0}+\delta\right]$.

(ii) Let $m=f\left(x_{0}-\delta\right)$ and $M=f\left(x_{0}+\delta\right)$. Then, since $f$ is strictly increasing on $\left[x_{0}-\delta, x_{0}+\delta\right]$, it follows that $m \leq f(x) \leq M$ for all $x \in\left[x_{0}-\delta, x_{0}+\delta\right]$. Since $f$ is $\mathcal{R}$-analytic on $\left[x_{0}-\delta, x_{0}+\delta\right]$, it follows that $f$ satisfies the intermediate value theorem on $\left[x_{0}-\delta, x_{0}+\delta\right]$; and hence $f\left(\left[x_{0}-\delta, x_{0}+\delta\right]\right)=[m, M]$. 
(iii) Define $g:[m, M] \rightarrow\left[x_{0}-\delta, x_{0}+\delta\right]$ as follows: given $y \in[m, M]$, then there exists a unique $x \in\left[x_{0}-\delta, x_{0}+\delta\right]$ such that $f(x)=y$; let $g(y)=x$. It follows that

$$
\begin{aligned}
& (g \circ f)(x)=g(f(x))=g(y)=x \text { for all } x \in\left[x_{0}-\delta, x_{0}+\delta\right] ; \text { and } \\
& (f \circ g)(y)=f(g(y))=f(x)=y \text { for all } y \in[m, M] .
\end{aligned}
$$

Thus, $g$ is the inverse of $f$ on $\left[x_{0}-\delta, x_{0}+\delta\right]$.

To show that $g$ is differentiable on $[m, M]$, let $y \in[m, M]$ be given and let $x=g(y)$. Let $\epsilon>0$ in $\mathcal{R}$ be given and let $\epsilon_{1} \in(0, \epsilon)$ be such that

$$
\left|\frac{f(t)-f(x)}{t-x}-f^{\prime}(x)\right|<\min \left\{\frac{\left|f^{\prime}(x)\right|}{2}, \frac{\epsilon\left|f^{\prime}(x)\right|^{2}}{2}\right\}
$$

for $t \in\left[x_{0}-\delta, x_{0}+\delta\right]$ satisfying $0<|t-x|<\epsilon_{1}$. It follows that

$$
|f(t)-f(x)|>\frac{\left|f^{\prime}(x)\right||t-x|}{2}
$$

when $t \in\left[x_{0}-\delta, x_{0}+\delta\right]$ and $0<|t-x|<\epsilon_{1}$. By Corollary 3.4, there exist $\zeta_{1}, \zeta_{2}>0$ such that $f\left(\left[x_{0}-\delta, x_{0}+\delta\right] \cap\left[x-\epsilon_{1} / 2, x+\epsilon_{1} / 2\right]\right)=\left[y-\zeta_{1}, y+\zeta_{2}\right]$. Let $\zeta=\min \left\{\zeta_{1}, \zeta_{2}\right\}$. Then $g((y-\zeta, y+\zeta)) \subset\left[x_{0}-\delta, x_{0}+\delta\right] \cap\left(x-\epsilon_{1}, x+\epsilon_{1}\right)$.

Now let $z \in[m, M]$ be such that $0<|z-y|<\zeta$; and let $t=g(z)$. Then

$$
|t-x|<\epsilon_{1}<\epsilon \text {. }
$$

It follows that

$$
\begin{aligned}
\left|\frac{g(z)-g(y)}{z-y}-\frac{1}{f^{\prime}(x)}\right| & =\left|\frac{t-x}{f(t)-f(x)}-\frac{1}{f^{\prime}(x)}\right| \\
& =\frac{|t-x|\left|\frac{f(t)-f(x)}{t-x}-f^{\prime}(x)\right|}{\left|f^{\prime}(x)\right||f(t)-f(x)|} \\
& <\frac{|t-x| \epsilon\left|f^{\prime}(x)\right|^{2} / 2}{\left|f^{\prime}(x)\right||f(t)-f(x)|} \\
& <\frac{|t-x| \epsilon\left|f^{\prime}(x)\right|^{2} / 2}{\left|f^{\prime}(x)\right|\left|f^{\prime}(x)\right||t-x| / 2}=\epsilon .
\end{aligned}
$$

Hence $g$ is differentiable at $y$, and $g^{\prime}(y)=1 / f^{\prime}(x)=1 / f^{\prime}(g(y))$.

Finally, if $f^{\prime}\left(x_{0}\right)<0$ then we apply the arguments above to $-f$ which is $\mathcal{R}$ analytic on $[a, b]$ and satisfies $(-f)^{\prime}\left(x_{0}\right)>0$.

We finish this paper with the following conjecture regarding the inverse function $g$ in Corollary 3.8.

Conjecture 3.9. Let $a<b$ in $\mathcal{R}$ be given, let $f:[a, b] \rightarrow \mathcal{R}$ be $\mathcal{R}$-analytic on $[a, b]$, and let $x_{0} \in(a, b)$ be such that $f^{\prime}\left(x_{0}\right) \neq 0$. Let $\delta, m, M$ and $g:[m, M] \rightarrow$ $\left[x_{0}-\delta, x_{0}+\delta\right]$ be as in Corollary 3.8. Then $g$ is $\mathcal{R}$-analytic on $[m, M]$.

\section{References}

[1] N. L. Alling. Foundations of Analysis over Surreal Number Fields. North Holland, 1987.

[2] M. Berz. Analysis on a nonarchimedean extension of the real numbers. Lecture Notes, 1992 and 1995 Mathematics Summer Graduate Schools of the German National Merit Foundation. MSUCL-933, Department of Physics, Michigan State University, 1994.

[3] M. Berz. Calculus and numerics on Levi-Civita fields. In M. Berz, C. Bischof, G. Corliss, and A. Griewank, editors, Computational Differentiation: Techniques, Applications, and Tools, pages 19-35, Philadelphia, 1996. SIAM. 
[4] W. Krull. Allgemeine Bewertungstheorie. J. Reine Angew. Math., 167:160-196, 1932.

[5] D. Laugwitz. Tullio Levi-Civita's work on nonarchimedean structures (with an Appendix: Properties of Levi-Civita fields). In Atti Dei Convegni Lincei 8: Convegno Internazionale Celebrativo Del Centenario Della Nascita De Tullio Levi-Civita, Academia Nazionale dei Lincei, Roma, 1975.

[6] T. Levi-Civita. Sugli infiniti ed infinitesimi attuali quali elementi analitici. Atti Ist. Veneto di Sc., Lett. ed Art., 7a, 4:1765, 1892.

[7] T. Levi-Civita. Sui numeri transfiniti. Rend. Acc. Lincei, 5a, 7:91,113, 1898.

[8] L. Neder. Modell einer Leibnizschen Differentialrechnung mit aktual unendlich kleinen Größen. Mathematische Annalen, 118:718-732, 1941-1943.

[9] S. Priess-Crampe. Angeordnete Strukturen: Gruppen, Körper, projektive Ebenen. Springer, Berlin, 1983.

[10] P. Ribenboim. Fields: Algebraically Closed and Others. Manuscripta Mathematica, 75:115150, 1992

[11] W. H. Schikhof. Ultrametric Calculus: An Introduction to p-Adic Analysis. Cambridge University Press, 1985.

[12] K. Shamseddine. New Elements of Analysis on the Levi-Civita Field. PhD thesis, Michigan State University, East Lansing, Michigan, USA, 1999. also Michigan State University report MSUCL-1147.

[13] K. Shamseddine. On the topological structure of the Levi-Civita field. J. Math. Anal. Appl., 368:281-292, 2010.

[14] K. Shamseddine and M. Berz. Exception handling in derivative computation with nonArchimedean calculus. In M. Berz, C. Bischof, G. Corliss, and A. Griewank, editors, Computational Differentiation: Techniques, Applications, and Tools, pages 37-51, Philadelphia, 1996. SIAM.

[15] K. Shamseddine and M. Berz. Convergence on the Levi-Civita field and study of power series. In Proc. Sixth International Conference on p-adic Functional Analysis, pages 283-299, New York, NY, 2000. Marcel Dekker.

[16] K. Shamseddine and M. Berz. Intermediate values and inverse functions on non-Archimedean fields. Int. J. Math. Math. Sci., 30:165-176, 2002.

[17] K. Shamseddine and M. Berz. Measure theory and integration on the Levi-Civita field. Contemp. Math., 319:369-387, 2003.

[18] K. Shamseddine and M. Berz. Analytical properties of power series on Levi-Civita fields. Ann. Math. Blaise Pascal, 12(2):309-329, 2005.

[19] K. Shamseddine and M. Berz. Generalized power series on a non-Archimedean field. Indag. Math., 17(3):457-477, 2006.

[20] K. Shamseddine and M. Berz. Intermediate value theorem for analytic functions on a LeviCivita field. Bull. Belg. Math. Soc. Simon Stevin, 14:1001-1015, 2007.

[21] K. Shamseddine and V. Zeidan. One-dimensional optimization on non-Archimedean fields. $J$. Nonlinear Convex Anal., 2:351-361, 2001.

[22] K. Shamseddine and V. Zeidan. Constrained second order optimization on non-Archimedean fields. Indag. Math., 14:81-101, 2003.

Department of Physics and Astronomy, University of Manitoba, Winnipeg, ManiTOBA R3T 2N2, CANADA

E-mail address: khodr@physics.umanitoba.ca 